Progress in Nuclear Science and Technology

Volume 6 (2019) pp. 225-229

\title{
ARTICLE
}

\section{ACE library of JENDL-4.0/HE}

\author{
Norihiro Matsuda $^{\mathrm{a}^{*}}$, Satoshi Kunieda ${ }^{\mathrm{a}}$, Tsutomu Okamoto ${ }^{\mathrm{a}, \mathrm{b}}$, Kenichi Tada ${ }^{\mathrm{a}}$ and Chikara Konno ${ }^{\mathrm{a}}$ \\ ${ }^{a}$ Japan Atomic Energy Agency, 2-4 Shirakata, Tokai-mura, Naka-gun, Ibaraki-ken, 319-1195, Japan; ${ }^{b}$ Nippon Advanced Information \\ Service, 416 Muramatsu, Tokai-mura, Naka-gun, Ibaraki-ken, 319-1112, Japan (Current affiliation)
}

\begin{abstract}
The Intra-Nuclear Cascade model employed into general-purpose Monte-Carlo simulation codes is not always applicable in the energy region from $20 \mathrm{MeV}$ to several hundreds of $\mathrm{MeV}$. In order to improve accuracy of the Monte-Carlo simulations including this energy region, the fourth version of Japanese Evaluated Nuclear Data Library High Energy File (JENDL-4.0/HE) as a new special purpose file, was released in 2015. It includes evaluated cross-sections for incident neutrons and protons up to $200 \mathrm{MeV}$ for 130 and 133 nuclei, respectively. A Compact version of ENDF (ACE) library of all the neutron- and proton-induced reaction data for the MCNP, MCNPX and PHITS codes has been produced with the nuclear data processing code NJOY2016.9, which was modified to keep laboratory angle-energy distribution form (LAW=67) in the ACE library of proton because the original NJOY converts laboratory angle-energy distribution form (LAW=67) to continuum energy-angle distribution form $(\mathrm{LAW}=61)$ automatically and PHITS can only treat angle-energy distribution form $(\mathrm{LAW}=67)$ for proton. Validations for the ACE library were performed through benchmark calculations for high-energy shielding experiments with PHITS.
\end{abstract}

Keywords: JENDL-4.0/HE; NJOY2016; ACE library; Monte Carlo; PHITS; validation

\section{Introduction}

The Intra-Nuclear Cascade (INC) model employed into general-purpose Monte-Carlo simulation codes (e.g. INCL4.6 [1] into PHITS [2]) is not always applicable in the energy region from typical upper limit of evaluated nuclear data $(20 \mathrm{MeV})$ to several hundreds of $\mathrm{MeV}$. Nuclear reactions in this energy region are much sensitive to nuclear structure, especially for light-nuclei such as Lithium and Beryllium. Theoretical estimations of the cross-sections are also difficult for these light-nuclei because of applicable limit of nuclear modeling code. Therefore, semi-classical approximation by INC model is not adequate below several hundreds of $\mathrm{MeV}$. In order to improve accuracy of the Monte-Carlo simulations including this energy region, a new special purpose file of Japanese Evaluated Nuclear Data Library, JENDL-4.0 High-Energy File (JENDL-4.0/HE) [3], was released in 2015. It includes evaluated cross-sections for incident neutrons and protons up to $200 \mathrm{MeV}$ for 130 and 133 nuclei, respectively. Substantial features of the JENDL-4.0/HE are, (1) systematic evaluation for medium-mass nuclei using the CCONE code [4] with recent progress in the optical model [5] and clustering pre-equilibrium model [6], (2) challenges to the evaluations of light-nuclei such for the $\mathrm{p}^{+6,7} \mathrm{Li}$ and $\mathrm{p}+{ }^{9} \mathrm{Be}$ reactions through the interpolation/extrapolation of experimental data, and (3) inheritance of the existing

*Corresponding author. Email: matsuda.norihiro@jaea.go.jp evaluated nuclear data of JENDL-4.0 [7] below $20 \mathrm{MeV}$ and JENDL/HE-2007 [8] which is a previous version of JENDL High-Energy File. The details of the evaluation methods and approaches about the JENDL-4.0/HE are summarized in the report [3].

We produced an ACE library of JENDL-4.0/HE by a nuclear data processing code NJOY [9]. The ACE library which is used in general-purpose Monte-Carlo simulation codes, such as MCNP [10], MCNPX [11] and PHITS, is a compact version of the Evaluated Nuclear Data File (ENDF) [12] library. The ENDF is a format to store and retrieve the evaluated nuclear data. For this purpose, several modifications were applied to the latest version of NJOY, NJOY2016.9. Validations of this ACE library were carried out by the analysis of high-energy shielding experiments with PHITS, and the results were summarized.

\section{Processing of evaluated nuclear data with NJOY}

The JENDL-4.0/HE has ENDF-6 format, which allows higher incident energies than $20 \mathrm{MeV}$. In the NJOY2016, coupled energy-angle distributions in File 6 of an evaluated nuclear data represented laboratory angle-energy law ( $\mathrm{LAW}=7$ format) are converted into the continuum energy-angle distribution form (ACE $\mathrm{LAW}=61$ format, named for ENDF File 6, Law 1) triggered by the newfor $=1$ option in NJOY's input which 
is now the default. Although the continuum energyangle representation (ACE LAW=61 format) is good for use with the Monte-Carlo simulation codes, PHITS can only treat laboratory angle-energy distribution form (ACE LAW=67 format, named for ENDF File 6, Law 7) for protons. Thus, modifications of NJOY2016 are needed in order to produce an ACE library of JENDL-4.0/HE. The original NJOY2016 was modified based on the report about processing of the JENDL/HE-2007 [13]. Processing procedures of JENDL-4.0/HE with the modified version of NJOY2016 are described below.

\subsection{Modifications of NJOY2016}

The ACER module in NJOY2016 was modified to produce the ACE library. The conversions from laboratory angle-energy law (ENDF $\mathrm{LAW}=7$ format) to continuum energy-angle distribution form (ACE $\mathrm{LAW}=61$ format) are triggered by the no $7=1$ flag of TOPFIL subroutine in ACER module. However, processing program relevant to no7 flag "off" was halted because of program error. To properly store the coupled energy-angle data into ACE library, new subroutine, ACECSD having a similar function to ACENSD, was prepared in ACELOD subroutine. The array size in the ACER module was also increased as necessary because the file size of each nucleus in JENDL-4.0/HE is larger than those in typical evaluated nuclear data below $20 \mathrm{MeV}$.

\subsection{Procedures to process ACE library}

All the nuclear data for neutrons of 130 nuclei and for protons of 133 nuclei in JENDL-4.0/HE were processed. The processing procedures differ between incident neutrons and protons.

\subsubsection{ACE-formatted neutron library}

In order to process the neutron-induced nuclear data, the MODER, RECONR, BROADR, HEATR, THERMR, GASPR, PURR, and ACER modules were used in order. For nuclei without unresolved resonance data, the PURR module was skipped. The basic conditions of NJOY processing for incident neutrons are summarized in Table 1.

Table 1. Basic conditions of NJOY processing for incident neutron nuclear data.

\begin{tabular}{cc}
\hline Condition & Input parameter \\
\hline Tolerance (Precision) & $0.1 \%$ \\
Temperature & $300 \mathrm{~K}$ \\
Upper boundary of thermal energy & $4.6 \mathrm{eV}$ \\
Treatment of Inelastic scattering & Free-gas model \\
in thermal energy region & \\
Unresolved resonance & Yes \\
Library identifier and suffix ID & $89 \mathrm{c}$ \\
\hline The ZAID for metastable nucleus, ${ }^{242 \mathrm{~m}} \mathrm{Am}$ is changed to \\
95292.89c to give the uniquely ZAID.
\end{tabular}

\subsubsection{ACE-formatted proton library}

For the incident proton nuclear data, the MODER, RECONR, and ACER modules were used in order. The basic conditions of NJOY processing for incident proton nuclear data are summarized in Table 2.

Table 2. Basic conditions of NJOY processing for incident proton nuclear data.

\begin{tabular}{cc}
\hline Condition & Input parameter \\
\hline Tolerance (Precision) & $0.1 \%$ \\
Temperature & $0 \mathrm{~K}$ \\
Library identifier and suffix ID & $89 \mathrm{~h}$ \\
\hline The ZAID for metastable nucleus, ${ }^{242 \mathrm{~m}} \mathrm{Am}$ is changed to \\
95292.89h to give the uniquely ZAID.
\end{tabular}

\section{V\&V of the ACE library and discussion}

\subsection{Verification of the ACE library}

Consistency checks for the produced ACE file were performed with the ACER and VIEWR modules in NJOY2016. The ACER module has a capability to read an ACE library and check the data for some common problems [14]. The VIEWR module can generate color Postscript files from an output file of the ACER module. The principal cross-sections: (1) total, (2) absorption, (3) elastic, and (4) photon production, shown in Figure 1 were visually inspected.
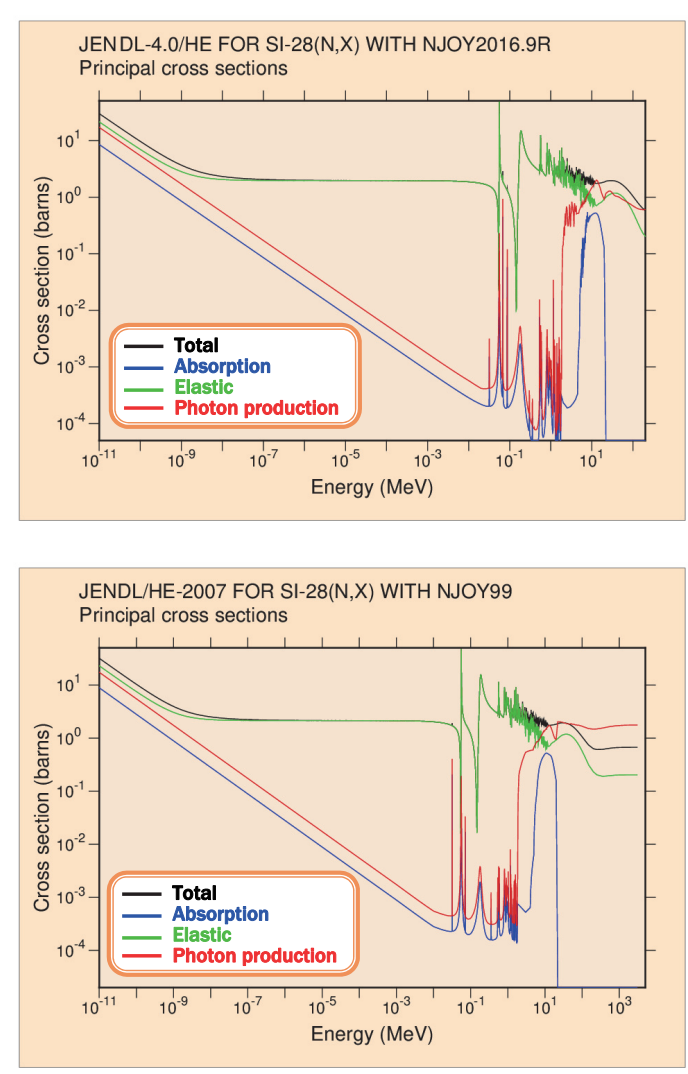

Figure 1. Principal cross-sections of $n+{ }^{28} \mathrm{Si}$ generated from the JENDL-4.0/HE (upper) and JENDL/HE-2007 (lower). 
The color Postscript files were also generated from the ACE library of JENDL/HE-2007 as a sterling example for the ACE library processed the evaluated high-energy cross-sections. Comparisons were conducted between the principal cross-sections of the JENDL-4.0/HE and those of JENDL/HE-2007. Some discrepancy observed in the figure arose from the differences of the evaluation processes between the JENDL/HE-2007 and the JENDL-4.0/HE.

\subsection{Validations of the ACE library}

Validations of the ACE library of JENDL-4.0/HE were carried out through benchmark calculations for neutron yields from the ${ }^{9} \mathrm{Be}(\mathrm{p}, \mathrm{xn})$ reactions at $10 \mathrm{MeV}$ [15] and for high-energy shielding experiments at TIARA (Takasaki Ion accelerator for Advanced Radiation Application) [16, 17] and RCNP (Research Center for Nuclear Physics, Osaka university) [18, 19] with PHITS version 2.82 .

The calculated results by PHITS with JENDL-4.0/HE were compared with the experimental data. Furthermore, the results were compared with those of the INCL4.6 into PHITS.

\subsubsection{Benchmark calculations for neutron yields}

Figure 2 shows double-differential cross-sections for $10-\mathrm{MeV}$ proton incidence on thin Beryllium target.

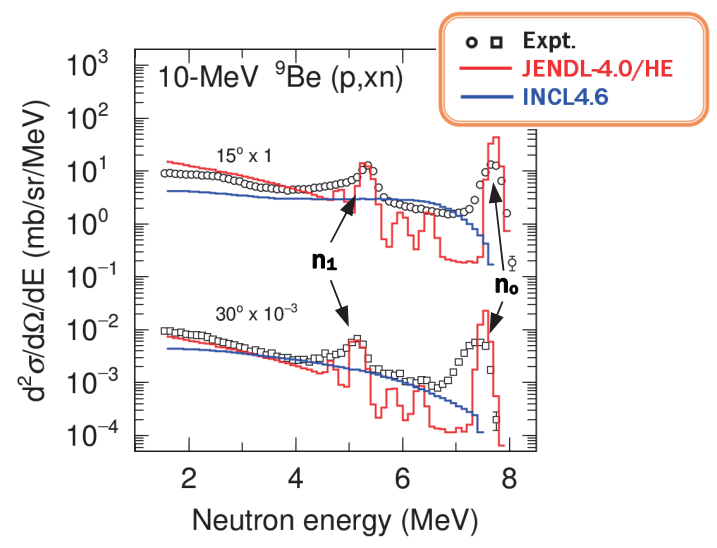

Figure 2. Double-differential cross-sections at $15^{\circ}$ and $30^{\circ}$. The experimental data (black) are compared with the calculated results by PHITS with JENDL-4.0/HE (red) and INCL4.6 in PHITS (blue).

The experimental ${ }^{9} \mathrm{Be}(\mathrm{p}, \mathrm{xn})$ neutron spectra exhibit two large peaks $\left(\mathrm{n}_{0}\right.$ and $\mathrm{n}_{1}$ ) corresponding to the nuclear structure. The calculated results by PHITS with JENDL-4.0/HE represented the two large peaks. However, the INCL4.6 could not reproduce such kind of peak. In the calculated results with JENDL-4.0/HE, two small peaks around $6 \mathrm{MeV}$ come from pseudo levels assumed in the evaluation process based on the unknown level structure of ${ }^{9} \mathrm{~B}[20]$. In the comparison between the experimental data and the calculated results with JENDL-4.0/HE, the small discrepancies observed in the peak regions around $8 \mathrm{MeV}$ are due to the experimental uncertainty of time-of-flight method.

\subsubsection{Benchmark calculations for shielding experiments}

The experimental geometries of the high-energy shielding experiments are shown in Figure 3. Quasimonoenergetic neutrons of 65 and $138 \mathrm{MeV}$ are generated by the ${ }^{7} \mathrm{Li}(\mathrm{p}, \mathrm{n})^{7} \mathrm{Be}$ reaction in TIARA and RCNP experiments, respectively. Since the INCL4.6 could not reproduce the quasi-monoenergetic peak from the ${ }^{7} \mathrm{Li}(\mathrm{p}, \mathrm{n})^{7} \mathrm{Be}$ reactions, the calculations were started from secondary neutrons which were generated at each target position, and with solid angles $\left(5.94 \times 10^{-4}\right.$, $3.13 \times 10^{-4} \mathrm{sr}$ ) due to the collimators. The tally size in the calculations reproduced the detector size in TIARA and RCNP experiments.
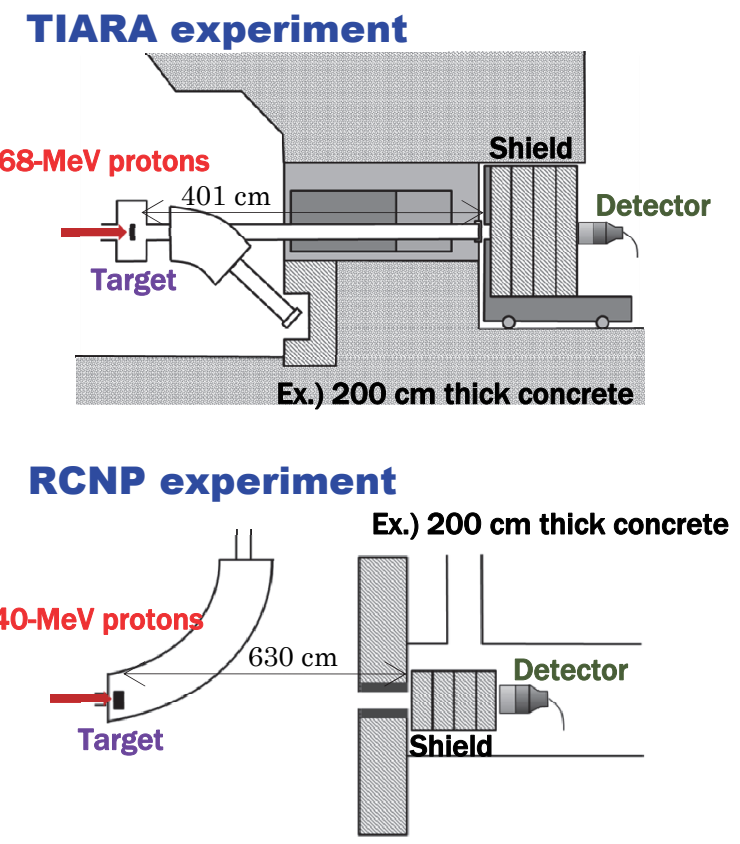

Figure 3. Experimental geometries of TIARA (upper) and RCNP (lower) experiments.

Figure 4 shows experimental data and calculated results by the PHITS with JENDL-4.0/HE and with the INCL4.6. The overestimations with the INCL4.6 of the TIARA experiment at 100 - and $150-\mathrm{cm}$-thick concrete are improved by using the PHITS with JENDL-4.0/HE. In the experimental data of TIARA experiment, the peak width around $60 \mathrm{MeV}$ is broader than that of the calculated result. They are due to the experimental uncertainty of the time-of-flight method.

For the benchmark calculation of RCNP experiment, there is no great distinction between the both calculation results (i.e., the INCL4.6 already has a good prediction accuracy for the incident energy region.).

Thorough the benchmark calculations, it was found that the accuracy of PHITS was improved by using the ACE library of JENDL-4.0/HE. We recommend using 
the JENDL-4.0/HE up to $200 \mathrm{MeV}$ instead of INC model.
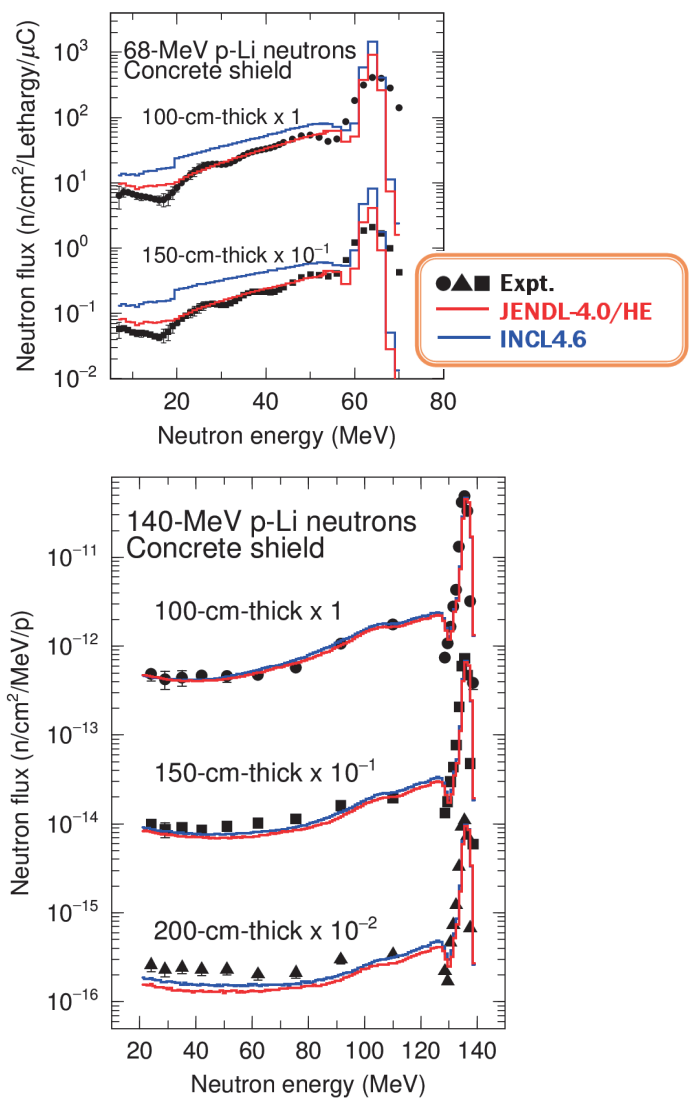

Figure 4. Experimental data and calculated results by PHITS with JENDL-4.0/HE (red) and INCL4.6 in PHITS (blue) at TIARA (upper) and RCNP (lower) experiments.

\section{Conclusion}

In order to improve the accuracy of Monte-Carlo simulations from 20 to $200 \mathrm{MeV}$, JENDL-4.0/HE was released. The ACE library of JENDL-4.0/HE was processed with the modified version of NJOY2016. The accuracy of the ACE library was confirmed through benchmark calculations. It was concluded that we recommend using the ACE library of JENDL-4.0/HE up to $200 \mathrm{MeV}$ instead of INC model.

\section{References}

[1] A. Boudard, J. Cugnon, J.-C. David, S. Leray and D. Mancusi, New potentialities of the Liege intranuclear cascade model for reactions induced by nucleons and light charged particles, Phys. Rev. C 87 (2013), p.014606.

[2] T. Sato, K. Niita, N. Matsuda, S. Hashimoto, Y. Iwamoto, S. Noda, T. Ogawa, H. Iwase, H. Nakashima, T. Fukahori, K. Okumura, T. Kai, S. Chiba, T. Furuta and L. Sihver, Particle and Heavy Ion Transport Code System PHITS, Version 2.52, J. Nucl. Sci. Technol. 50 (2013), pp. 913-923.
[3] S. Kunieda, O. Iwamoto, N. Iwamoto, F. Minato, T. Okamoto, T. Sato, H. Nakashima, Y. Iwamoto, H. Iwamoto, F. Kitatani, T. Fukahori, Y. Watanabe, N. Shigyo, S. Chiba, N. Yamamoto, M. Hagiwara, K. Niita, K. Kosako, S. Hirayama and T. Murata, Proc. the 2015 Symposium on Nuclear Data, November 19-20, 2015, Ibaraki Quantum Beam Research Center, Tokai-mura, Ibaraki, Japan, (Overview of JENDL-4.0/HE and benchmark calculations), JAEA-Conf 2016-004, Japan Atomic Energy Agency, (2016), pp. 41-46.

[4] O. Iwamoto, N. Iwamoto, S. Kunieda, F. Minato and K. Shibata, The CCONE code system and its application to nuclear data evaluation for fission and other reactions, Nuclear Data Sheets 131 (2016), pp. 259-288.

[5] S. Kunieda, S. Chiba, K. Shibata, A. Ichihara and E. Sh. Sukhovitski, Coupled-channels optical model analyses of nucleon-induced reactions for medium and heavy nuclei in the energy region from $1 \mathrm{keV}$ to $200 \mathrm{MeV}$, J. Nucl. Sci. and Technol. 44 (2007), pp. 838-852.

[6] S. Kunieda, R.C. Haight, T. Kawano, M.B. Chadwick, S.M. Sterbenz, F.B. Bateman, O.A. Wasson, S.M. Grimes, P. Maier-Komor, H. Vonach, T. Fukahori and Y. Watanabe, Measurement and model analysis of $(\mathrm{n}, \mathrm{x} \alpha)$ cross sections for $\mathrm{Cr}, \mathrm{Fe},{ }^{56} \mathrm{Co}$, and ${ }^{58,60} \mathrm{Ni}$ from threshold energy to $150 \mathrm{MeV}$, Phys. Rev. C85 (2012), p.054602.

[7] K. Shibata, O. Iwamoto, T. Nakagawa, N. Iwamoto, A. Ichihara, S. Kunieda, S. Chiba, K. Furutaka, N. Otsuka, T. Ohasawa, T. Murata, H. Matsunobu, A. Zukeran, S. Kamada and J. Katakura, JENDL-4.0: A new library for nuclear science and engineering, J. Nucl. Sci. and Technol. 48 (2011), pp. 1-30.

[8] Y. Watanabe, K. Kosako, S. Kunieda, S. Chiba, R. Fujimoto, H. Harada, M. Kawai, F. Maekawa, T. Murata, H. Nakashima, K. Niita, N. Shigyo, S. Shimakawa, N. Yamano and T. Fukahori, Status of JENDL high energy file, Proc. Int. Conf. on Nuclear Data for Science and Technology (ND2010), Jeju Island, Korea, April 26-30, 2010, J. Kor. Phys. Soc. 59 (2011), pp. 1040-1045.

[9] R.E. MacFarlane, D.W. Muir, R.M. Boicourt, A.C. Kahler and J.L. Conlin, The NJOY Nuclear Data Processing System, Version 2016, LA-UR-1720093, Los Alamos National Laboratory, (2016).

[10]X-5 Monte Carlo Team, MCNP - A general $N$-Particle transport code, Version 5: Volume I: Overview and theory, LA-UR-03-1987, Los Alamos National Laboratory, (2003).

[11]D.B. Pelowitz, J.W. Durkee, J.S. Elson, M.L. Fensin, J.S. Hendricks, M.R. James, R.C. Johns, G.W. McKinney, S.G. Mashnik, J.M. Verbeke, L.S. Waters and T.A. Wilcox, MCNPX 2.7.0 extensions, LA-UR-11-02295, Los Alamos National Laboratory, (2011).

[12]A. Trkov and M. Herman (Ed.), ENDF-6 Formats Manual: Data Formats and Procedures for the 
Evaluated Nuclear Data Files: ENDF/B-VI and ENDF/B-VII, BNL-90365-2009, National Nuclear Data Center, Brookhaven National Laboratory, (2009).

[13]T. Sasa, T. Sugawara, K. Kosako and T. Fukahori, Continuous Energy Cross Section Library for $M C N P / M C N P X$ based on JENDL High Energy File 2007, JAEA-Data/Code 2008-022, Japan Atomic Energy Agency, (2008).

[14]R.E. Macfarlane and A.C. Kahler, Methods for Processing ENDF/B-VII with NJOY, LA-UR-1004652, Los Alamos National Laboratory, (2010).

[15]Y. Iwamoto, Y. Sakamoto, N. Matsuda, Y. Nakane, K. Ochiai, H. Kaneko, K. Niita, T. Shibata and H. Nakashima, Measurements of double-differential neutron-production cross-sections for the ${ }^{9} \mathrm{Be}(\mathrm{p}, \mathrm{xn})$ and ${ }^{9} \mathrm{Be}(\mathrm{d}, \mathrm{xn})$ reactions at $10 \mathrm{MeV}, \mathrm{Nucl}$. Inst. and Methods Phys. Res. A 598 (2009), pp.687-695.

[16]N. Nakao, H. Nakashima, T. Nakamura, Sh. Tanaka, Su. Tanaka, K. Shin, M. Baba, Y. Sakamoto and Y. Nakane, Transmission through shields of quasi-monoenergetic neutrons generated by 43 - and $68-\mathrm{MeV}$ Protons -I: Concrete shielding experiment and calculation for practical application, Nucl. Sci. Eng. 124 (1996), pp. 228-242.

[17]H. Nakashima, N. Nakao, Sh. Tanaka, T. Nakamura, K. Shin, Su. Tanaka, H. Takada, S. Meigo, Y. Nakane, Y. Sakamoto and M. Baba, Transmission through shields of quasimonoenergetic neutrons generated by 43- and
68-MeV Protons -II: Iron shielding experiment and analysis for investigating calculational method and cross-section data, Nucl. Sci. Eng. 124 (1996), pp. 243-257.

[18]H. Yashima, H. Iwase, M. Hagiwara, Y. Kirihara, S. Taniguchi, H. Yamakawa, K. Oishi, Y. Iwamoto, D. Satoh, Y. Nakane, H. Nakashima, T. Itoga, N. Nakao, T. Nakamura, A. Tamii and K. Hatanaka, Benchmark experiment of neutron penetration through iron and concrete shields for hundreds-of-MeV quasi-monoenergetic neutrons-I: Measurements of neutron spectrum by a multimoderator spectrometer, Nucl. Tecnol. 168 (2008), pp. 298-303.

[19]M. Hagiwara, H. Iwase, Y. Kirihara, H. Yashima, Y. Iwamoto, D. Satoh, Y. Nakane, H. Nakashima, T. Nakamura, A. Tamii and K. Hatanaka, Benchmark experiment of neutron penetration through iron and concrete shields for hundreds-of-MeV quasi-monoenergetic neutronsII: Measurements of neutron spectrum by an organic liquid scintillator, Nucl. Tecnol. 168 (2008), pp. 304-309.

[20]J.K. Tuli, Evaluated Nuclear Structure Data File (ENSDF): A manual for preparation of data sets, National Nuclear Data Center, Brookhaven National Laboratory (BNL), BNL-NCS-51655-01/ 02-Rev. (2001). http://www.nndc.bnl.gov/ensdf/index.jsp 